\title{
Metabolic and adipose risk factors for NIDDM and coronary disease in third-generation Japanese-American men and women with impaired glucose tolerance
}

\author{
W. Y. Fujimoto ${ }^{1}$, R.W. Bergstrom ${ }^{1}$, D.L.Leonetti ${ }^{2}$, L. L. Newell-Morris ${ }^{2}$, W. P.Shuman ${ }^{3}$, P. W. Wahl ${ }^{4}$ \\ ${ }^{1}$ Department of Medicine, University of Washington, Seattle, Washington, USA \\ ${ }^{2}$ Department of Anthropology, University of Washington, Seattle, Washington, USA \\ ${ }^{3}$ Department of Radiology, University of Washington, Seattle, Washington, USA \\ ${ }^{4}$ Department of Biostatistics, University of Washington, Seattle, Washington, USA
}

Summary Since second-generation (Nisei) Japanese Americans are prone to develop the insulin resistance syndrome, younger third-generation (Sansei) Japanese Americans from a cross-sectional $10 \%$ volunteer sample of Sansei men $(n=115)$ and women $(n=115)$ 34 years or older in King County, Washington with normal glucose tolerance or IGT were examined for metabolic and adipose risk factors associated with this syndrome. After an overnight 10-h fast, blood samples were taken for measurement of glucose, insulin, C-peptide, lipids, and lipoproteins, followed by a $3-\mathrm{h} 75$-g oral glucose tolerance test with blood samples taken for glucose, insulin, and C-peptide measurement. BMI $\left(\mathrm{kg} / \mathrm{m}^{2}\right)$, skinfolds, and body fat areas (by computed tomography) were measured. IGT was diagnosed in $19 \%$ of the men and $31 \%$ of the women. Men with IGT had more adiposity, both overall and in thoracic and visceral sites, had higher fasting plasma insulin and C-peptide, and tended to have higher fasting triglyceride and lower HDL cholesterol than men with normal glucose tolerance. Women with IGT had more thoracic subcutaneous fat and intra-abdominal fat and lower fasting HDL cholesterol than women with normal glucose tolerance, and tended to have higher fasting triglyceride and LDL cholesterol. Women with IGT also had higher fasting plasma insulin than women with normal glucose tolerance but tended to be less hyperinsulinaemic than men. Differences in fasting insulin, C-peptide, and lipids were best predicted by intra-abdominal fat. Thus metabolic (higher fasting insulin and a tendency to higher triglyceride and lower HDL cholesterol) and adipose (visceral adiposity) risk factors associated with the insulin resistance syndrome are identifiable among Sansei men and women with IGT, who may therefore be at increased risk of future development of NIDDM and CHD. [Diabetologia (1994) 37: 524-532]

Key words Impaired glucose tolerance, lipids, insulin, C-peptide, fat distribution, insulin resistance syndrome.
Previous research has shown a high prevalence of both NIDDM and CHD among second-generation (Nisei) Japanese-American men and a high prevalence of

Received: 9 July 1993

and in revised form: 14 December 1993

Corresponding author: Dr. W. Y. Fujimoto, Department of Medicine, Division of Metabolism, Endocrinology, and Nutrition, Mail Stop RG-26, University of Washington School of Medicine, Seattle, WA 98195, USA

Abbreviations: NIDDM, Non-insulin-dependent diabetes mellitus; CHD, coronary heart disease; IGT, impaired glucose tolerance; AUC, area under the curve; ANOVA, analysis of variance
NIDDM among Nisei women compared to native Japanese [1-5]. We have proposed that these observations reflect the propensity in Japanese Americans to develop the insulin resistance syndrome, defined as the clustering of insulin resistance, hyperinsulinaemia, obesity (especially central or visceral obesity), hypertension, hypertriglyceridaemia, and low HDL cholesterol [6-8]. The relationship of visceral obesity to the abnormalities of glucose and lipid metabolism have been previously reported [9-12]. Exeptions related to ethnicity have been reported, however, for certain features of this syndrome. For example, Saad et al. [13] have reported that plasma insulin levels and insulin resistance were related to blood pressure in Whites but not in Pima Indians or Blacks, although in another 
study of young black men a direct relationship between insulin resistance and blood pressure was observed [14]. Fasting insulin has been reported to be significantly correlated with blood pressure independent of obesity and age in Japanese children [15]. Studies in other migrant Asian populations have also shown that central adiposity and insulin resistance are associated with NIDDM and cardiovascular risk [16]. It would therefore be informative to know whether the younger third generation (Sansei) have any of the metabolic and adipose risk factors associated with the insulin resistance syndrome and the relationship of these to glucose tolerance status.

\section{Subjects, materials, and methods}

The protocol for this research was reviewed by the Human Subjects Review Committee at the University of Washington and each subject gave informed and signed consent. Subjects were enrolled through a community-wide recruitment using a comprehensive mailing list and telephone directory that included nearly $95 \%$ of the Japanese-American population of King County, Washington. Sansei were defined as any Japanese person born in the continental United States of Nisei parents or of one Nisei parent and one Sansei parent. The strategy was to study approximately $10 \%$ of the Sansei population 34 years old or older who were non-diabetic, and 230 subjects $(115$ men, 115 women) were enrolled from among 533 volunteers (266 men, 267 women) who met the criteria for generation and age. Excluded were 151 men and 152 women for the following reasons: same gender as enrolled sibling (14 men, 21 women) or enrolled first cousin ( 23 men, 37 women), born and raised in $\mathrm{Ha}$ waii ( 29 men, 26 women), educated in Japan ( 3 men, 2 women), Nisei parents were educated in Japan (15 men, 11 women), moved out of King County (13 men, 4 women), adopted (1 man, 3 women), scheduling conflicts ( 22 men, 20 women), excess volunteers ( 23 men, 9 women), and confounding medical conditions (11 men, 19 women). These medical conditions were insulin-dependent diabetes mellitus or NIDDM ( 7 men, 3 women), cancer ( 2 men, 3 women), hepatitis ( 1 man), kidney disease ( 2 women), thyroid disease ( 2 women), lupus (1 woman), steroid-treated asthma (2 women), pregnancy (1 woman), pulmonary disease (1 woman), Epstein-Barr virus infection (1 woman), and refusal to discontinue nasal decongestants (1 man, 3 women).

Subjects were studied at the General Clinical Research Center, University of Washington Medical Center following an overnight 10 -h fast. All subjects underwent a 75 -g oral glucose tolerance test, and glucose tolerance was categorized according to the criteria of the World Health Organization [17]. Baseline blood samples were withdrawn for measurement of glucose, insulin, Cpeptide, lipids, and lipoproteins. Subjects then ingested a solution of glucose $(75 \mathrm{~g})$ in water over approximately $1 \mathrm{~min}$ and blood samples were withdrawn at $30,60,120$, and $180 \mathrm{~min}$ for measurement of plasma glucose, insulin, and C-peptide. AUC for insulin, C-peptide, and glucose were calculated using the trapezoidal rule.

Height was measured to the nearest tenth centimeter and weight to the nearest hundredth kilogram and BMI was calculated as weight $(\mathrm{kg}) /$ height $^{2}\left(\mathrm{~m}^{2}\right)$. Skinfold thickness $(\mathrm{mm})$ was measured (Lange calipers) on the left side of the body at six anatomic sites: forearm, biceps, triceps, subscapula, abdomen (midway between the umbilicus and the most lateral point), and ante- rior thigh. A midaxillary site was measured only in men. Crosssectional body fat areas $\left(\mathrm{cm}^{2}\right)$ were assessed by computed tomography. Single slices were obtained at three levels: thorax at the level of the nipples, abdomen at the level of the umbilicus, and left midthigh; areas of subcutaneous thoracic, abdominal, and thigh fat and of intra-abdominal fat within the confines of the transversalis fascia were calculated, as described previously [18].

Plasma glucose was measured by an automated glucose oxidase method at the University of Washington Medical Center, Clinical Chemistry Laboratory. Plasma insulin and C-peptide were measured by radioimmunoassay performed in the Radioimmunoassay Core of the Diabetes Endocrinology Research Center using primary antisera developed in the Core [19, 20]. All lipid and lipoprotein measurements were performed at the Northwest Lipid Research Laboratory, according to modified procedures of the Lipid Research Clinics [21]. Apoproteins $A 1$ and $B$ were determined by standardized radioimmunoassays [22].

\section{Statistical analysis}

All values are expressed as mean \pm SEM. For variables with highly skewed distributions, geometric means are presented and statistical tests computed on logarithmic transformations. Risk factors were analysed using normal glucose tolerance and IGT as the categorical variable. Statistically significant differences between groups were analysed by applying the two-sample $t$ test; all tests were two-sided. Adjustments (BMI, height, weight, intra-abdominal fat, and age) were made by analysis of covariance. In some analyses, subjects were separated into tertiles of the independent variable of interest, with the upper tertile further divided into an upper and a lower half, and data were analysed by ANOVA with tests for linear, cubic, and quadratic trends. Glucose, insulin, and C-peptide values during the oral glucose tolerance test were analysed by repeated measures ANOVA.

\section{Results}

Clinical characteristics: the mean age and age range were virtually identical between Sansei men and women (mean, 40.2 \pm 0.4 years and range, 34.0-51.3 years, and mean, $40.1 \pm 0.4$ and range, 34.0-53.1 years, respectively). Among the men, 93 had normal glucose tolerance and $22(19.1 \%)$ had IGT, while 79 women had normal glucose tolerance and $36(31.3 \%)$ had IGT. The mean age of men with IGT $(42.0 \pm 1.1$ years) was significantly greater than that of men with normal glucose tolerance $(39.6 \pm 0.4$ years, $p=0.049)$, whereas the mean age of women with IGT ( $39.8 \pm 0.5$ years) was not significantly different from that of women with normal glucose tolerance $(40.8 \pm 0.7$ years, $p=0.25)$.

\section{Adiposity and body fat distribution by glucose tolerance} status and gender: data concerning mean BMI, skinfolds, and body fat areas by computed tomography are summarized in Table 1. Men with IGT had greater mean BMI than men with normal glucose tolerance. After adjustment for BMI, only the computed tomography measurements of subcutaneous thoracic and intra-abdominal fat remained significantly different 
Table 1. BMI and body fat distribution by glucose tolerance status (normal and IGT) and gender

\begin{tabular}{|c|c|c|c|c|c|c|}
\hline & \multicolumn{3}{|l|}{ Men } & \multicolumn{3}{|l|}{ Women } \\
\hline & $\begin{array}{l}\text { Normal } \\
(n=93)\end{array}$ & $\begin{array}{l}\text { IGT } \\
(n=22)\end{array}$ & $p$-value & $\begin{array}{l}\text { Normal } \\
(n=79)\end{array}$ & $\begin{array}{l}\text { IGT } \\
(n=36)\end{array}$ & $p$-value \\
\hline $\mathrm{BMI}\left(\mathrm{kg} / \mathrm{m}^{2}\right)$ & $24.5 \pm 0.3$ & $26.8 \pm 0.9$ & 0.021 & $22.5 \pm 0.3$ & $23.3 \pm 0.7$ & 0.28 \\
\hline \multicolumn{7}{|l|}{ Skinfolds (mm): } \\
\hline $\begin{array}{l}\text { Forearm }^{\mathrm{a}} \\
\text { Biceps }^{\mathrm{a}} \\
\text { Triceps }^{\mathrm{a}} \\
\text { Subscapula } \\
\text { Chest } \\
\text { Abdomen } \\
\text { Thigh }\end{array}$ & $\begin{array}{r}4.9 \pm 0.2 \\
7.5 \pm 0.5 \\
12.1 \pm 0.6 \\
20.6 \pm 0.9 \\
28.7 \pm 1.3^{\mathrm{b}} \\
26.9 \pm 1.2 \\
22.3 \pm 1.6\end{array}$ & $\begin{array}{r}5.8 \pm 0.6 \\
11.0 \pm 2.0 \\
15.9 \pm 2.0 \\
27.8 \pm 2.2 \\
34.8 \pm 3.0 \\
34.2 \pm 3.0 \\
30.3 \pm 3.8\end{array}$ & $\begin{array}{l}0.56 \\
0.060 \\
0.027 \\
0.001 \\
0.049 \\
0.012 \\
0.037\end{array}$ & $\begin{array}{r}7.9 \pm 0.4 \\
13.5 \pm 0.9 \\
21.8 \pm 0.9 \\
21.0 \pm 1.3 \\
31.1 \pm 1.2 \\
45.0 \pm 1.3\end{array}$ & $\begin{array}{r}8.6 \pm 1.1 \\
15.5 \pm 1.4 \\
23.3 \pm 1.3 \\
25.0 \pm 2.2 \\
\\
34.1 \pm 2.4 \\
45.3 \pm 1.3\end{array}$ & $\begin{array}{l}0.87 \\
0.20 \\
0.29 \\
0.10 \\
\\
\\
0.27 \\
0.86\end{array}$ \\
\hline \multicolumn{7}{|c|}{ Computed tomography fat area $\left(\mathrm{cm}^{2}\right)$ : } \\
\hline $\begin{array}{l}\text { Thoracic subcutaneous } \\
\text { Abdominal subcutaneous } \\
\text { Intra-abdominal }^{\mathrm{a}} \\
\text { Thigh subcutaneous }\end{array}$ & $\begin{array}{r}70.8 \pm 3.9 \\
135.4 \pm 7.2 \\
67.8 \pm 3.7 \\
48.3 \pm 2.2\end{array}$ & $\begin{aligned} 113.7 & \pm 13.3 \\
184.2 & \pm 17.8 \\
104.1 & \pm 11.8 \\
56.4 & \pm 5.5\end{aligned}$ & $\begin{array}{l}0.005 \\
0.005 \\
0.006 \\
0.13\end{array}$ & $\begin{array}{r}88.4 \pm 7.6 \\
152.7 \pm 8.7 \\
37.6 \pm 2.5 \\
94.0 \pm 3.9\end{array}$ & $\begin{array}{c}123.7 \pm 16.2 \\
175.8 \pm 16.3 \\
57.7 \pm 7.4 \\
95.1 \pm 6.8\end{array}$ & $\begin{array}{l}0.027 \\
0.18 \\
0.008 \\
0.88\end{array}$ \\
\hline
\end{tabular}

${ }^{\text {a }}$ Statistical tests computed on logarithmic transformation

${ }^{\mathrm{b}} n=90$ due to missing values in three subjects

Values are shown as mean \pm SEM

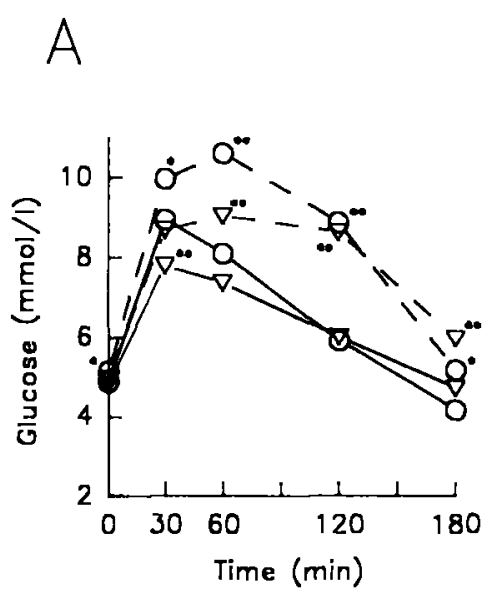

Fig. 1A-C. Plasma glucose (A), insulin (B), and C-peptide(C) levels during a 3-h 75-g oral glucose tolerance test for men $(O)$ and women $(\nabla)$ with normal glucose tolerance $(-)$ and IGT (---). ${ }^{*} p<0.05,{ }^{* *} p<0.001$ for comparisons between normal glucose tolerance and IGT within each gender

between glucose tolerance groups $(p=0.014$ and $p=0.024$, respectively).

By contrast, the mean BMI of women did not differ significantly by glucose tolerance category. Significant differences in subcutaneous thoracic fat and intra-abdominal fat by computed tomography remained, after adjustment for BMI in both women and men $(p=0.018$ and $p=0.013$, respectively).

Insulin, $C$-peptide, and glucose levels by glucose tolerance status and gender (Fig. 1): both fasting insulin $(p=0.004)$ and fasting C-peptide levels $(p<0.001)$ were much higher in men with IGT than those with normal glucose tolerance. Differences were no longer sig-

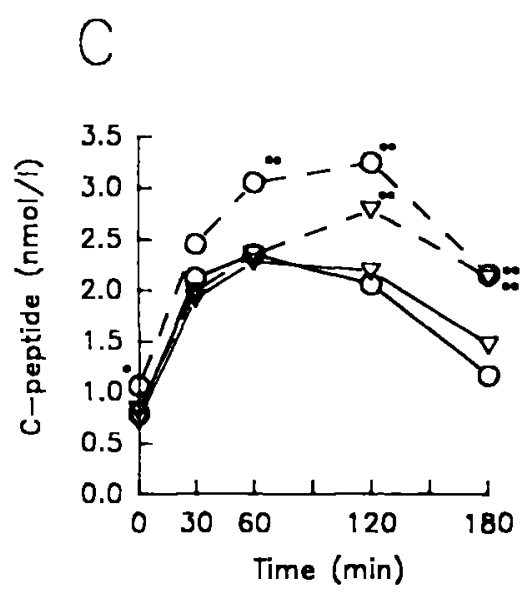

nificant, however, after simultaneous adjustment for age, BMI, and intra-abdominal fat $(p=0.095$ for insulin and $p=0.21$ for C-peptide). The greatest effect upon the $p$-value was observed with adjustment for intra-abdominal fat. Fasting insulin was also significantly higher in women with IGT than those with normal glucose tolerance $(p=0.027)$, and again the difference did not remain significant after simultaneous adjustment for age, BMI, and intra-abdominal fat $(p=0.39)$. This effect was mainly attributable to intra-abdominal fat. Fasting C-peptide levels did not differ significantly between women with IGT and those with normal glucose tolerance $(p=0.12)$.

The peak insulin and C-peptide levels were delayed and higher in men and women with IGT than in those with normal glucose tolerance (repeated measures ANOVA: $p<0.001$ for insulin by glucose tolerance status in men and $p=0.027$ in women, $p<0.001$ for Cpeptide by glucose tolerance status in men and women, $p<0.001$ for interaction between glucose tolerance status and sample time for both insulin and C-peptide 
Table 2. Fasting plasma lipids, lipoproteins, and apoproteins by glucose tolerance status (normal and IGT) and gender

\begin{tabular}{|c|c|c|c|c|c|c|}
\hline & \multicolumn{3}{|l|}{ Men } & \multicolumn{3}{|l|}{ Women } \\
\hline & $\begin{array}{l}\text { Normal } \\
(n=93)\end{array}$ & $\begin{array}{l}\text { IGT } \\
(n=22)\end{array}$ & $p$-value & $\begin{array}{l}\text { Normal } \\
(n=79)\end{array}$ & $\begin{array}{l}\text { IGT } \\
(n=36)\end{array}$ & $p$-value \\
\hline \multicolumn{7}{|l|}{ Cholesterol $(\mathrm{mmol} / \mathrm{l})$} \\
\hline $\begin{array}{l}\text { Total } \\
\text { LDL } \\
\text { HDL } \\
\text { Triglycerides }(\mathrm{mmol} / \mathrm{l})^{\mathrm{a}}\end{array}$ & $\begin{array}{l}5.64 \pm 0.09 \\
3.60 \pm 0.09 \\
1.37 \pm 0.04 \\
1.64 \pm 0.16\end{array}$ & $\begin{array}{l}5.80 \pm 0.25 \\
3.47 \pm 0.18 \\
1.23 \pm 0.07 \\
2.95 \pm 0.73\end{array}$ & $\begin{array}{l}0.55 \\
0.51 \\
0.066 \\
0.079\end{array}$ & $\begin{array}{l}5.17 \pm 0.08 \\
3.00 \pm 0.06 \\
1.75 \pm 0.05 \\
1.05 \pm 0.09\end{array}$ & $\begin{array}{l}5.33 \pm 0.16 \\
3.25 \pm 0.12 \\
1.56 \pm 0.06 \\
1.41 \pm 0.24\end{array}$ & $\begin{array}{l}0.40 \\
0.073 \\
0.048 \\
0.055\end{array}$ \\
\hline \multicolumn{7}{|l|}{ Apoproteins (mg/dl): } \\
\hline $\begin{array}{l}\text { Apo-A1 } \\
\text { Apo-B }\end{array}$ & $\begin{array}{l}141 \pm 3 \\
110 \pm 3\end{array}$ & $\begin{array}{l}132 \pm 4 \\
110 \pm 4\end{array}$ & $\begin{array}{l}0.11 \\
0.93\end{array}$ & $\begin{array}{r}155 \pm 3 \\
91 \pm 2\end{array}$ & $\begin{array}{r}152 \pm 5 \\
98 \pm 4\end{array}$ & $\begin{array}{l}0.60 \\
0.078\end{array}$ \\
\hline
\end{tabular}

${ }^{a}$ Statistical tests computed on logarithmic transformations

Values are shown as mean \pm SEM

Table 3. Metabolic variables by intra-abdominal fat area tertiles in men

\begin{tabular}{|c|c|c|c|c|c|c|c|}
\hline & $\begin{array}{l}\text { Lower third } \\
(n=38)\end{array}$ & $\begin{array}{l}\text { Middle third } \\
(n=39)\end{array}$ & $\begin{array}{l}\text { Upper third, } \\
\text { lower half } \\
(n=19)\end{array}$ & $\begin{array}{l}\text { Upper third, } \\
\text { upper half } \\
(n=19)\end{array}$ & $p$-value & $\begin{array}{l}p \text {-value } \\
\text { (linear } \\
\text { trend) }\end{array}$ & $\begin{array}{l}p \text {-value (ad- } \\
\text { justed for BMI, } \\
\text { weight, height) }\end{array}$ \\
\hline $\begin{array}{l}\text { Fasting insulin } \\
(\mathrm{pmol} / \mathrm{l})\end{array}$ & $\begin{array}{l}56.2 \pm 4.0 \\
(62.8)^{c}\end{array}$ & $\begin{array}{l}80.4 \pm 5.2 \\
(81.4)\end{array}$ & $\begin{array}{l}83.7 \pm 8.5 \\
(82.4)\end{array}$ & $\begin{array}{l}138.5 \pm 17.8 \\
(124.4)\end{array}$ & $<0.0001$ & $\begin{array}{l}<0.0001 \\
C: 0.029\end{array}$ & $<0.001$ \\
\hline $\begin{array}{l}\text { Insulin AUC } \\
(\mathrm{nmol} / 1 \times \min )\end{array}$ & $\begin{array}{l}45.7 \pm 3.1 \\
(48.7)\end{array}$ & $\begin{array}{l}71.7 \pm 5.7 \\
(72.2)\end{array}$ & $\begin{array}{l}72.4 \pm 19.7 \\
(71.8)\end{array}$ & $\begin{array}{l}139.2 \pm 24.7 \\
(130.9)\end{array}$ & $<0.0001$ & $<0.0001$ & $<0.001$ \\
\hline $\begin{array}{l}\text { Fasting C-peptide } \\
(\mathrm{nmol} / \mathrm{l})\end{array}$ & $\begin{array}{l}0.66 \pm 0.03 \\
(0.72)\end{array}$ & $\begin{array}{l}0.81 \pm 0.04 \\
(0.82)\end{array}$ & $\begin{array}{l}0.85 \pm 0.05 \\
(0.84)\end{array}$ & $\begin{array}{l}1.10 \pm 0.08 \\
(1.01)\end{array}$ & $<0.0001$ & $\begin{array}{l}<0.0001 \\
\text { C: } 0.014\end{array}$ & 0.001 \\
\hline $\begin{array}{l}\text { C-peptide AUC } \\
(\text { nmol } / 1 \times \min )\end{array}$ & $\begin{array}{l}303 \pm 9 \\
(313)\end{array}$ & $\begin{array}{l}353 \pm 16 \\
(355)\end{array}$ & $\begin{array}{l}363 \pm 17 \\
(362)\end{array}$ & $\begin{array}{l}471 \pm 34 \\
(456)\end{array}$ & $<0.0001$ & $<0.0001$ & $<0.001$ \\
\hline $\begin{array}{l}\text { Fasting glucose } \\
(\mathrm{mmol} / \mathrm{l})\end{array}$ & $\begin{array}{l}4.86 \pm 0.08 \\
(4.94)\end{array}$ & $\begin{array}{l}4.98 \pm 0.08 \\
(4.99)\end{array}$ & $\begin{array}{l}5.14 \pm 0.11 \\
(5.13)\end{array}$ & $\begin{array}{l}5.27 \pm 0.10 \\
(5.18)\end{array}$ & 0.019 & 0.0017 & 0.38 \\
\hline 2-h glucose $(\mathrm{mmol} / \mathrm{l})$ & $\begin{array}{l}6.36 \pm 0.26 \\
(6.32)\end{array}$ & $\begin{array}{l}6.22 \pm 0.26 \\
(6.24)\end{array}$ & $\begin{array}{l}7.27 \pm 0.38 \\
(7.29)\end{array}$ & $\begin{array}{l}7.36 \pm 0.40 \\
(7.38)\end{array}$ & 0.022 & 0.0085 & 0.039 \\
\hline $\begin{array}{l}\text { Glucose AUC } \\
(\mathrm{mmol} / 1 \times \mathrm{min})\end{array}$ & $\begin{array}{l}1219 \pm 34 \\
(1214)\end{array}$ & $\begin{array}{l}1241 \pm 34 \\
(1245)\end{array}$ & $\begin{array}{l}1367 \pm 54 \\
(1369)\end{array}$ & $\begin{array}{l}1374 \pm 41 \\
(1374)\end{array}$ & 0.012 & 0.0020 & 0.032 \\
\hline $\begin{array}{l}\text { Total cholesterol } \\
(\mathrm{mmol} / \mathrm{l})\end{array}$ & $\begin{array}{l}5.60 \pm 0.15 \\
(5.51)\end{array}$ & $\begin{array}{l}5.38 \pm 0.12 \\
(5.39)\end{array}$ & $\begin{array}{l}6.18 \pm 0.24 \\
(6.20)\end{array}$ & $\begin{array}{l}6.07 \pm 0.24 \\
(6.13)\end{array}$ & 0.0040 & $\begin{array}{l}0.0096 \\
\text { C:0.016 }\end{array}$ & 0.003 \\
\hline $\begin{array}{l}\text { LDL-cholesterol } \\
(\mathrm{mmol} / \mathrm{)})\end{array}$ & $\begin{array}{l}3.43 \pm 0.12 \\
(3.34)\end{array}$ & $\begin{array}{l}3.40 \pm 0.11 \\
(3.39)\end{array}$ & $\begin{array}{l}4.00 \pm 0.23 \\
(4.01)\end{array}$ & $\begin{array}{l}3.92 \pm 0.22 \\
(4.00)\end{array}$ & 0.011 & 0.0050 & 0.005 \\
\hline $\begin{array}{l}\text { HDL-cholesterol } \\
(\mathrm{mmol} / \mathrm{l})\end{array}$ & $\begin{array}{l}1.55 \pm 0.06 \\
(1.46)\end{array}$ & $\begin{array}{l}1.34 \pm 0.05 \\
(1.34)\end{array}$ & $\begin{array}{l}1.23 \pm 0.06 \\
(1.24)\end{array}$ & $\begin{array}{l}1.10 \pm 0.03 \\
(1.18)\end{array}$ & $<0.0001$ & $<0.0001$ & 0.024 \\
\hline $\begin{array}{l}\text { Triglycerides } \\
(\mathrm{mmol} / \mathrm{l})\end{array}$ & $\begin{array}{l}2.70 \pm 0.37 \\
(3.07)\end{array}$ & $\begin{array}{l}3.19 \pm 0.76 \\
(3.26)\end{array}$ & $\begin{array}{l}3.93 \pm 1.8 \\
(3.87)\end{array}$ & $\begin{array}{l}4.60 \pm 0.91 \\
(4.01)\end{array}$ & 0.015 & 0.0013 & 0.47 \\
\hline $\begin{array}{l}\text { Apoprotein A-1 } \\
\text { (mg/dl) }\end{array}$ & $\begin{array}{l}150.1 \pm 4.8 \\
(144.6)\end{array}$ & $\begin{array}{l}135.6 \pm 3.4) \\
(135.8)\end{array}$ & $\begin{array}{l}136.3 \pm 4.6 \\
(137.2)\end{array}$ & $\begin{array}{l}127.6 \pm 4.0 \\
(132.0)\end{array}$ & 0.0046 & 0.0010 & 0.34 \\
\hline $\begin{array}{l}\text { Apoprotein B } \\
(\mathrm{mg} / \mathrm{dl})\end{array}$ & $\begin{array}{l}102.7 \pm 3.2 \\
(102.1)\end{array}$ & $\begin{array}{l}105.1 \pm 3.3 \\
(105.1)\end{array}$ & $\begin{array}{l}118.5 \pm 5.7 \\
(118.6)\end{array}$ & $\begin{array}{l}124.3 \pm 5.8 \\
(124.8)\end{array}$ & 0.0012 & 0.0001 & 0.005 \\
\hline
\end{tabular}

a Trend is linear unless indicated ( $\mathrm{C}$ : cubic)

${ }^{b}$ Geometric means are presented and statistics are computed on natural logarithms
${ }^{c}$ Mean adjusted for BMI, weight, and height is shown in parentheses

Values are shown as mean $\pm \mathbf{S E M}$ in men and women). The differences in insulin and Cpeptide levels by glucose tolerance status remained significant after adjusting for age, BMI, and intra-abdominal fat in men. Differences in insulin levels by glucose tolerance status were, however, not significant $(p=0.33)$ in women after adjusting for age, BMI, and intra-abdominal fat, while differences in C-peptide levels by glucose tolerance status remained significant $(p=0.021)$. In general, men with IGT tended to have higher insulin and C-peptide levels than women with 
Table 4. Metabolic variables by intra-abdominal fat area tertiles in women

\begin{tabular}{|c|c|c|c|c|c|c|c|}
\hline & $\begin{array}{l}\text { Lower third } \\
(n=38)\end{array}$ & $\begin{array}{l}\text { Middle third } \\
(n=39)\end{array}$ & $\begin{array}{l}\text { Upper third, } \\
\text { lower half } \\
(n=19)\end{array}$ & $\begin{array}{l}\text { Upper third, } \\
\text { upper half } \\
(n=19)\end{array}$ & $p$-value & $\begin{array}{l}p \text {-value } \\
\text { (linear } \\
\text { trend) }^{a}\end{array}$ & $\begin{array}{l}p \text {-value (ad- } \\
\text { justed for BMI, } \\
\text { weight, height) }\end{array}$ \\
\hline $\begin{array}{l}\text { Fasting insulin } \\
\text { (pmol/l) }\end{array}$ & $\begin{array}{l}57.4 \pm 5.2 \\
(65.5)^{\mathrm{c}}\end{array}$ & $\begin{array}{l}60.6 \pm 2.8 \\
(64.8)\end{array}$ & $\begin{array}{l}88.6 \pm 9.5 \\
(85.3)\end{array}$ & $\begin{array}{l}127.5 \pm 15.6 \\
(108.1)\end{array}$ & $<0.0001$ & $\begin{array}{l}<0.0001 \\
Q: 0.040\end{array}$ & 0.015 \\
\hline $\begin{array}{l}\text { Insulin } \mathrm{AUC}^{\mathrm{b}} \\
(\mathrm{nmol} / \mathrm{l} \times \mathrm{min})\end{array}$ & $\begin{array}{l}60.7 \pm 4.1 \\
(61.1)\end{array}$ & $\begin{array}{l}63.8 \pm 3.5 \\
(63.3)\end{array}$ & $\begin{array}{l}87.1 \pm 10.0 \\
(88.2)\end{array}$ & $\begin{array}{l}130.4 \pm 28.4 \\
(128.8)\end{array}$ & $<0.0001$ & $\begin{array}{l}<0.0001 \\
Q: 0.0057\end{array}$ & $<0.001$ \\
\hline $\begin{array}{l}\text { C-peptide AUC } \\
(\mathrm{nmol} / / \times \mathrm{min})\end{array}$ & $\begin{array}{l}316 \pm 11 \\
(308)\end{array}$ & $\begin{array}{l}343 \pm 9 \\
(335)\end{array}$ & $\begin{array}{l}384 \pm 14 \\
(392)\end{array}$ & $\begin{array}{l}451 \pm 28 \\
(464)\end{array}$ & $<0.0001$ & $<0.0001$ & $<0.001$ \\
\hline $\begin{array}{l}\text { Fasting glucose } \\
(\mathrm{mmol} / \mathrm{l})\end{array}$ & $\begin{array}{l}4.80 \pm 0.07 \\
(4.89)\end{array}$ & $\begin{array}{l}4.82 \pm 0.07 \\
(4.87)\end{array}$ & $\begin{array}{l}5.21 \pm 0.16 \\
(5.20)\end{array}$ & $\begin{array}{l}5.16 \pm 0.13 \\
(5.03)\end{array}$ & $<0.0001$ & 0.0010 & 0.16 \\
\hline $\begin{array}{l}\text { Total cholesterol } \\
(\mathrm{mmol} / \mathrm{l})\end{array}$ & $\begin{array}{l}4.97 \pm 0.12 \\
(5.07)\end{array}$ & $\begin{array}{l}5.29 \pm 0.11 \\
(5.34)\end{array}$ & $\begin{array}{l}5.31 \pm 0.19 \\
(5.31)\end{array}$ & $\begin{array}{l}5.68 \pm 0.22 \\
(5.52)\end{array}$ & 0.016 & 0.0022 & 0.42 \\
\hline $\begin{array}{l}\text { LDL-cholesterol } \\
(\mathrm{mmol} / \mathrm{l})\end{array}$ & $\begin{array}{l}2.79 \pm 0.08 \\
(2.81)\end{array}$ & $\begin{array}{l}3.10 \pm 0.09 \\
(3.11)\end{array}$ & $\begin{array}{l}3.29 \pm 0.17 \\
(3.31)\end{array}$ & $\begin{array}{l}3.48 \pm 0.15 \\
(3.44)\end{array}$ & 0.0003 & $<0.0001$ & 0.024 \\
\hline $\begin{array}{l}\text { HDL-cholesterol } \\
(\mathrm{mmol} / \mathrm{l})\end{array}$ & $\begin{array}{l}1.88 \pm 0.06 \\
(1.94)\end{array}$ & $\begin{array}{l}1.81 \pm 0.06 \\
(1.83)\end{array}$ & $\begin{array}{l}1.48 \pm 0.09 \\
(1.47)\end{array}$ & $\begin{array}{l}1.36 \pm 0.06 \\
(1.29)\end{array}$ & $<0.0001$ & $<0.0001$ & $<0.001$ \\
\hline $\begin{array}{l}\text { Triglycerides } \\
(\mathrm{mmol} / \mathrm{l})\end{array}$ & $\begin{array}{l}1.66 \pm 0.11 \\
(1.66)\end{array}$ & $\begin{array}{l}2.12 \pm 0.19 \\
(2.13)\end{array}$ & $\begin{array}{l}2.67 \pm 0.47 \\
(2.70)\end{array}$ & $\begin{array}{l}3.49 \pm 1.06 \\
(3.44)\end{array}$ & $<0.0001$ & $<0.0001$ & 0.002 \\
\hline $\begin{array}{l}\text { Apoprotein A-1 } \\
\text { (mg/dl) }\end{array}$ & $\begin{array}{l}160.3 \pm 4.2 \\
(163.3)\end{array}$ & $\begin{array}{l}161.7 \pm 3.8 \\
(162.8)\end{array}$ & $\begin{array}{l}138.0 \pm 5.0 \\
(137.7)\end{array}$ & $\begin{array}{l}141.9 \pm 5.7 \\
(138.1)\end{array}$ & 0.0005 & $\begin{array}{l}0.0005 \\
C: 0.017\end{array}$ & 0.003 \\
\hline
\end{tabular}

a Trend is linear unless indicated ( $\mathrm{C}$ : cubic or Q: quadratic)

${ }^{b}$ Geometric means are presented and statistics are computed on natural logarithms

${ }^{\mathrm{c}}$ Mean adjusted for BMI, weight, and height is shown in parentheses

Values are shown as mean $\pm \mathrm{SEM}$

IGT $(p<0.005$ for both insulin and C-peptide for interaction between glucose tolerance status and gender).

Lipids and lipoproteins by glucose tolerance status and gender: mean fasting lipids, lipoproteins, and apoproteins are shown in Table 2. Both normal glucose tolerant and IGT men had more atherogenic profiles than women. Within each gender, however, normal glucose tolerant and IGT subjects did not differ significantly although those with IGT tended to have higher triglyceride and lower HDL cholesterol levels (for women the difference in HDL cholesterol was significant, $p=0.048$ ). In addition, women with IGT tended to have higher LDL cholesterol and apoprotein B levels than did women with normal glucose tolerance. For all of these tendencies, $p$-values increased to more than 0.10 after simultaneously adjusting for age, BMI, and intraabdominal fat, and the greatest effect upon the $p$-value was seen with adjustment for intra-abdominal fat.

Relationship of adiposity to metabolic variables: men and women were separately divided into tertiles of several selected adiposity variables: BMI, as a measurement of general adiposity, and intra-abdominal fat area, thoracic subcutaneous fat area, abdominal subcutaneous fat area, and subscapular skinfold thickness as measurements of central adiposity. The uppermost tertile was further subdivided into a lower and an upper half. Mean values were calculated for insulin (fasting and AUC), C-peptide (fasting and AUC), glucose (fasting, 2-h, and AUC), cholesterol (total, LDL, and HDL), triglycerides, apoprotein A-1, and apoprotein $B$. The results with respect to intra-abdominal fat are shown in Table 3 for men and Table 4 for women.

All of the metabolic variables showed a significant relationship to intra-abdominal fat in both men and women. For all variables except fasting glucose, triglycerides, and apoprotein A-1 in men and fasting glucose, 2-h glucose, and total cholesterol in women, the relationships remained significant after adjusting for BMI, weight, and height.

Several metabolic variables were not significantly associated with the other adiposity variables. These 
Table 5. Glucose, lipids, and lipoproteins by fasting insulin tertiles in men and women

\begin{tabular}{|c|c|c|c|c|c|c|}
\hline & Lower third & Middle third & $\begin{array}{l}\text { Upper third, } \\
\text { lower half }\end{array}$ & $\begin{array}{l}\text { Upper third, } \\
\text { upper half }\end{array}$ & $p$-value & $\begin{array}{l}p \text {-value } \\
\text { (linear } \\
\text { trend) }\end{array}$ \\
\hline Men & $\mathrm{n}=43$ & $n=33$ & $n=19$ & $n=20$ & & \\
\hline Fasting glucose (mmol/l) & $4.87 \pm 0.07$ & $4.86 \pm 0.08$ & $5.16 \pm 0.09$ & $5.44 \pm 0.10$ & $<0.0001$ & $<0.0001$ \\
\hline 2-h glucose $(\mathrm{mmol} / \mathrm{l})$ & $6.62 \pm 0.17$ & $5.94 \pm 0.27$ & $6.52 \pm 0.29$ & $7.89 \pm 0.40$ & 0.0006 & $\begin{array}{l}0.012 \\
\mathrm{Q}: 0.0008\end{array}$ \\
\hline Glucose AUC $(\mathrm{mmol} / 1 \times \mathrm{min})$ & $1251 \pm 36$ & $1193 \pm 36$ & $1299 \pm 32$ & $1449 \pm 37$ & 0.0002 & $\begin{array}{l}0.0007 \\
Q: 0.0049\end{array}$ \\
\hline Total cholesterol (mmol/1) & $5.60 \pm 0.14$ & $5.60 \pm 0.15$ & $5.93 \pm 0.24$ & $5.83 \pm 0.24$ & 0.51 & \\
\hline LDL-cholesterol (mmol/l) & $3.48 \pm 0.12$ & $3.66 \pm 0.14$ & $3.80 \pm 0.21$ & $3.51 \pm 0.20$ & 0.52 & \\
\hline HDL-cholesterol (mmol/l) & $1.51 \pm 0.06$ & $1.37 \pm 0.04$ & $1.18 \pm 0.04$ & $1.13 \pm 0.06$ & $<0.0001$ & $<0.0001$ \\
\hline Triglycerides (mmol/l) & $2.75 \pm 0.25$ & $2.88 \pm 0.34$ & $4.21 \pm 0.67$ & $5.00 \pm 2.19$ & 0.0009 & 0.0001 \\
\hline Apoprotein A-1 (mg/dl) & $148.8 \pm 4.2$ & $140.8 \pm 3.8$ & $136.5 \pm 4.5$ & $122.6 \pm 4.3$ & 0.0030 & 0.0003 \\
\hline Apoprotein B (mg/dl) & $105.5 \pm 4.1$ & $111.6 \pm 5.9$ & $116.3 \pm 5.0$ & $109.1 \pm 5.0$ & 0.37 & \\
\hline Women & $\mathrm{n}=\mathbf{3 8}$ & $n=33$ & $n=23$ & $n=20$ & & \\
\hline Fasting glucose $(\mathrm{mmol} / \mathrm{l})$ & $4.70 \pm 0.07$ & $4.88 \pm 0.08$ & $5.09 \pm 0.11$ & $5.28 \pm 0.12$ & 0.0002 & $<0.0001$ \\
\hline 2-h glucose $(\mathrm{mmol} / \mathrm{l})$ & $6.66 \pm 0.22$ & $6.56 \pm 0.23$ & $7.25 \pm 0.37$ & $7.80 \pm 0.36$ & 0.015 & 0.0037 \\
\hline Glucose AUC $(\mathrm{mmol} / \mathrm{l} \times \mathrm{min})$ & $1180 \pm 30$ & $1225 \pm 38$ & $1292 \pm 57$ & $1381 \pm 48$ & 0.0089 & 0.0008 \\
\hline Total cholesterol (mmol/l) & $5.05 \pm 0.11$ & $5.18 \pm 0.13$ & $5.51 \pm 0.20$ & $5.43 \pm 0.20$ & 0.11 & \\
\hline LDL-cholesterol (mmol/l) & $2.89 \pm 0.08$ & $3.04 \pm 0.10$ & $3.33 \pm 0.16$ & $3.26 \pm 0.15$ & 0.028 & 0.0055 \\
\hline HDL-cholesterol (mmol/l) & $1.84 \pm 0.07$ & $1.72 \pm 0.06$ & $1.67 \pm 0.10$ & $1.48 \pm 0.08$ & 0.014 & 0.0015 \\
\hline Triglycerides (mmol/l) & $1.73 \pm 0.09$ & $2.10 \pm 0.30$ & $2.60 \pm 0.33$ & $3.09 \pm 1.02$ & 0.0004 & $<0.0001$ \\
\hline Apoprotein A-1 (mg/dl) & $158.0 \pm 4.0$ & $154.1 \pm 3.9$ & $156.4 \pm 7.0$ & $144.9 \pm 5.6$ & 0.32 & \\
\hline Apoprotein B (mg/dl) & $87.3 \pm 2.7$ & $91.2 \pm 3.2$ & $101.7 \pm 5.5$ & $98.2 \pm 4.6$ & 0.036 & 0.0098 \\
\hline
\end{tabular}

a Trend is linear unless indicated (Q: quadratic).

Values are shown as mean \pm SEM

data are not shown but the relationships that were not significant are summarized here. In men, total cholesterol and LDL cholesterol were not significantly related to BMI, thoracic subcutaneous fat, abdominal subcutaneous fat, and subscapular skinfold; triglycerides, apoprotein $B$, and $2-h$ glucose were not significantly related to subscapular skinfold; and glucose AUC was not significantly related to abdominal subcutaneous fat. In women, total cholesterol and apoprotein B were not significantly related to BMI and subscapular skinfold; apoprotein A-1 was not signficantly related to thoracic subcutaneous fat, abdominal subcutaneous fat, and subscapular skinfold; HDL-cholesterol was not significantly related to abdominal subcutaneous fat; and LDL-cholesterol was not significantly related to subscapular skinfold.

Relationship of fasting insulin and C-peptide to glucose, lipids, and lipoproteins: men and women were separately divided into tertiles of fasting insulin and C-peptide. The uppermost tertile was further subdivided into a lower half and an upper half. Mean values were calculated for glucose (fasting, 2-h, and AUC), cholesterol (total, LDL, and HDL), triglycerides, apoprotein A-1, and apoprotein $\mathrm{B}$. The results with respect to fasting insulin are shown in Table 5 for men and women.

All variables, except for total cholesterol, LDL-cholesterol, and apoprotein B in men and total cholesterol and apoprotein A-1 in women, were significantly related to fasting insulin. The relationship of fasting $\mathrm{C}$ peptide to these variables was similar in men. In women, however, total cholesterol $(p=0.041)$ and apoprotein A-1 $(p=0.0046)$ were also significantly related to fasting $\mathrm{C}$-peptide.

\section{Discussion}

Cross-sectional [23-26] and prospective [27] studies in Nisei men have identified greater adiposity, including greater amounts of thoracic and intra-abdominal fat, higher fasting plasma insulin, C-peptide, and triglyceride levels, and lower fasting plasma HDL cholesterol levels as some of the risk factors associated in this population with NIDDM and CHD. There is now much evidence that obesity, especially increased deposition of fat in a truncal location, is associated with hyperinsulinaemia and with NIDDM $[6-8,27,28]$. It is 
also clear that under certain conditions, fasting hyperinsulinaemia, measured as elevation of either insulin or C-peptide, reflects the presence of insulin resistance $[29,30]$. Although the prevalence of hyperinsulinaemia is increased in the non-diabetic members of populations with a high prevalence of NIDDM $[29,31]$ and insulin resistance is an early abnormality in subjects destined to develop NIDDM [27, 32-35], decompensation to NIDDM in insulin-resistant individuals may be prevented by the secretion of more insulin [36]. The resultant hyperinsulinaemia, however, may have adverse cardiovascular consequences [37-40].

The presence of IGT may be associated with increased risk of NIDDM, CHD, and hypertension [4147], and our results suggest that many Sansei men with IGT have begun to accumulate truncal and visceral fat and exhibit insulin resistance. Although Sansei women with IGT also had more truncal and intra-abdominal fat and higher fasting insulin levels than did women with normal glucose tolerance, levels were not as high as in Sansei men. It may be that the premenopausal status of the vast majority of the Sansei women in this sample is important. Indirect evidence in support of this conclusion is provided by the observation that Nisei women, the vast majority of whom are postmenopausal, have proportionally more intra-abdominal and thoracic subcutaneous fat and less thigh subcutaneous fat than do Sansei women [48]. It is also possible that for Sansei women, IGT may have been over-diagnosed because of the emphasis placed on the 120-min plasma glucose level. This possibility is suggested by the observation that the 30 -min and the 60 -min plasma glucose levels in women with IGT were much lower than in men with IGT.

In contrast to the marked differences in BMI, pattern of body fat distribution, and fasting insulin and Cpeptide levels between normal and IGT men, there were no significant lipid, lipoprotein, or apoprotein differences, although IGT men tended to have lower HDL cholesterol and higher triglyceride. IGT seemed to be more strongly associated with an atherogenic lipid profile among women, with significantly lower HDL cholesterol whereas triglyceride and LDL cholesterol both tended to be slightly higher. Analyses by tertiles of BMI or body fat distribution, however, showed significant relationships of all of the metabolic variables with intra-abdominal fat in both men and women as well as significant relationships for most of the metabolic variables with BMI, thoracic subcutaneous fat, abdominal subcutaneous fat, and subscapular skinfold. Furthermore, although Sansei women with IGT had lower fasting insulin levels than men with IGT, analyses by tertiles of fasting insulin and C-peptide, both of which were used as surrogate markers of insulin resistance, showed a significant relationship to glucose and most of the lipid and lipoprotein variables in both men and women. These results suggest that significant adverse changes in the metabolic variables are generally associated with adiposity, particularly with visceral adiposity, and with insulin resistance in both men and women, but that an association of these metabolic changes with glucose intolerance may require more severe glucose intolerance (e.g. diabetes). These possibilities may be clarified by prospective studies.

We have previously shown that when Nisei men and women are subdivided into either android or gynoid subsamples based upon distribution of subcutaneous fat, differences in intra-abdominal fat, and the fasting plasma levels of insulin, C-peptide, triglyceride, and HDL cholesterol between the fat pattern groups were highly significant for women but not for men. These observations suggest that in Nisei women but not in men, the android pattern of subcutaneous fat distribution is significantly related to greater metabolic and atherogenic risks [26]. We have also shown in a general linear model that the contributions of diabetic status (normal vs NIDDM) and intra-abdominal fat to potentially atherogenic variables (fasting insulin, triglyceride, and HDL cholesterol levels) differ between Nisei men and women [25]. For women, diabetic status did not substantially improve the model once intra-abdominal fat had been entered. By contrast in men, diabetic status remained a significant predictor for the metabolic variables even after intra-abdominal fat was considered in the model. Future prospective studies in Sansei women may show whether metabolic risk factors increase significantly at the time of menopause when truncal and visceral adiposity also increase.

In conclusion, metabolic and adipose risk factors associated with the insulin resistance syndrome are identifiable among Sansei men and women with IGT, who may therefore be at increased risk of developing NIDDM and CHD. Among women, fasting insulin and visceral adiposity were not as increased as in men. Whether this finding is due to their premenopausal hormonal status may be elucidated by prospective studies.

Acknowledgements. We gratefully acknowledge the skilled assistance of staff members, especially Ms. J.Shofer for her assistance with the statistical analyses. We are grateful to the King County Japanese-American community for their support and cooperation, and the many individuals who volunteered to participate in the study. This research was supported by National Institutes of Health Grant R22 DK 31170 and by facilities and services of the Diabetes Endocrinology Research Center (P30 DK 17047), the Clinical Nutrition Research Unit (P30 DK 35816), the University of Washington Medical Center General Clinical Research Center (MO1 RR 00037), and the Northwest Lipid Research Center Lipoprotein Laboratory.

\section{References}

1. Fujimoto WY, Leonetti DL, Kinyoun JL et al. (1987) Prevalence of diabetes mellitus and impaired glucose tolerance among second-generation Japanese-American men. Diabetes 36: 721-729 
2. Fujimoto WY, Leonetti DL, Kinyoun JL, Shuman WP, Stolov WC, Wahl PW (1987) Prevalence of complications among second-generation Japanese-American men with diabetes, impaired glucose tolerance, and normal glucose tolerance. Diabetes 36: 730-739

3. Fujimoto WY, Leonetti DL, Bergstrom RW, Kinyoun JL, Stolov WC, Wahl PW (1991) Glucose intolerance and diabetic complications among Japanese-American women. Diab Res Clin Prac 13: 119-130

4. Fujimoto WY, Akanuma Y, Kanazawa Y, Mashiko S, Leonetti D, Wahl P (1989) Plasma insulin levels in Japanese and Japanese-American men with type 2 diabetes may be related to the occurrence of cardiovascular disease. Diab Res Clin Prac 6: 121-127

5. Fujimoto WY, Bergstrom RW, Newell-Morris L, Leonetti DL (1989) Nature and nurture in the etiology of type 2 diabetes mellitus in Japanese Americans. Diabetes Metab Rev 5: 607-625

6. Reaven GM (1988) Role of insulin resistance in human disease. Diabetes 37: 1595-1607

7. Kaplan NM (1989) The deadly quartet: upper-body obesity, glucose intolerance, hypertriglyceridemia, and hypertension. Arch Intern Med 149: 1514-1520

8. DeFronzo RA, Ferrannini E (1991) Insulin resistance: a multifaceted syndrome responsible for NIDDM, obesity, hypertension, dyslipidemia and atherosclerotic cardiovascular disease. Diabetes Care 14: 173-194

9. Fujioka S, Matsuzawa Y, Tokunaga K, Tarui S (1987) Contribution of intra-abdominal fat accumulation to the impairment of glucose and lipid metabolism in human obesity. Metabolism 36: 54-59

10. Peiris AN, Sothmann MS, Hoffmann RG et al. (1989) Adiposity, fat distribution, and cardiovascular risk. Ann Intern Med 110: 867-872

11. Peiris AN, Sothmann MS, Hennes MI et al. (1989) Relative contribution of obesity and body fat distribution to alterations in glucose insulin homeostasis: predictive values of selected indices in premenopausal women. Am J Clin Nutr 49: 758-764

12. Despres JP, Moorjani S, Lupien PJ, Tremblay A, Nadeau A, Bouchard C (1990) Regional distribution of body fat, plasma lipoproteins, and cardiovascular disease. Arteriosclerosis 10: 497-511

13. Saad M, Lillioja S, Nyomba B et al. (1991) Racial differences in the relation between blood pressure and insulin resistance. New Engl J Med 324: 733-739

14. Falkner B, Hulman S, Tannenbaum J, Kushner H (1990) Insulin resistance and blood pressure in young black men. Hypertension 16: 706-711

15. Kanai H, Matsuzawa Y, Tokunaga K et al. (1990) Hypertension in obese children: fasting serum insulin levels are closely correlated with blood pressure. Int J Obesity 14: 1047-1056

16. McKeigue PM, Shah B, Marmot MG (1991) Relation of central obesity and insulin resistance with diabetes and cardiovascular risk in South Asians. Lancet 337: 382-386

17. World Health Organization Expert Committee on Diabetes Mellitus (1980) Second report. Technical Report Series 646. World Health Organization, Geneva

18. Shuman WP, Newell Morris LL, Leonetti DL et al. (1986) Abnormal body fat distribution detected by computed tomography in diabetic men. Invest Radiol 21: 483-487

19. Morgan DR, Lazarow A (1963) Immunoassay of insulin: two antibody system. Diabetes 12: 115-126

20. Ward WK, Paquette TL, Frank BH, Porte D Jr (1986) A sensitive radioimmunoassay for human proinsulin with sequential use of antisera to C-peptide and insulin. Clin Chem 32: $728-733$
21. Warnick GR (1986) Enzymatic methods for quantification of lipoproteins. Meth Enzymol 129: 101-123

22. Marcovina SM, Albers JJ, Dati F, Ledue TB, Ritchie RF (1991) International Federation of Clinical Chemistry standardization project for the measurement of apolipoproteins A-1 and B. Clin Chem 37: 1676-1682

23. Newell-Morris L, Treder RP, Shuman WP, Fujimoto WY (1989) Fatness, fat distribution and glucose tolerance in second-generation Japanese American (Nisei) men. Am J Clin Nutr 50: 9-18

24. Bergstrom RW, Leonetti DL, Newell-Morris LL, Shuman WP, Wahl PW, Fujimoto WY (1990) Association of plasma triglyceride and C-peptide with coronary heart disease in Japanese-American men with a high prevalence of glucose intolerance. Diabetologia 33: 489-496

25. Fujimoto WY, Newell-Morris LL, Grote M, Bergstrom RW, Shuman WP (1991) Visceral fat obesity and morbidity: NIDDM and atherogenic risk in Japanese American men and women. Int J Obesity 15 [Suppl 2]: 41-44

26. Fujimoto WY, Newell-Morris LL, Shuman WP (1991) Intraabdominal fat and risk variables for non-insulin-dependent diabetes (NIDDM) and coronary heart disease in Japanese American women with android or gynoid fat patterning. In: Oomura Y, Tarui S, Inoue S, Shimazu T (eds) Progress in obesity research 1990 . John Libbey, London, pp 317-322

27. Bergstrom RW, Newell-Morris LL, Leonetti DL, Shuman WP, Wahl PW, Fujimoto WY (1990) Association of elevated fasting C-peptide and increased intra-abdominal fat distribution with development of NIDDM in Japanese-American men. Diabetes 39: 104-111

28. Peiris AN, Mueller RA, Smith GA, Struve MF, Kissebah AH (1986) Splanchnic insulin metabolism in obesity: influence of body fat distribution. J Clin Invest 78: 1648-1657

29. Lillioja S, Mott DM, Zawadzki JK et al. (1987) In vivo insulin action is familial characteristic in nondiabetic Pima Indians. Diabetes 36: 1329-1335

30. Laakso M (1993) How good a marker is insulin level for insulin resistance? Am J Epidemiol 137: 959-965

31. Haffner SM, Stern MP, Hazuda HP, Pugh JA, Patterson JK (1986) Hyperinsulinemia in a high risk population for noninsulin-dependent diabetes. New Engl J Med 315: 220-224

32. Sicree RA, Zimmet PZ, King HOM, Coventry JS (1987) Plasma insulin in Nauruans: prediction of deterioration in glucose tolerance over 6 years. Diabetes 36: 179-186

33. Haffner SM, Stern MP, Mitchell BD, Hazuda HP, Patterson JK (1990) Incidence of type II diabetes in Mexican Americans predicted by fasting insulin and glucose levels, obesity, and body-fat distribution. Diabetes 39: 281-288

34. Warram JH, Martin BC, Krolewski AS, Soeldner JS, Kahn CR (1990) Slow glucose removal rate and hyperinsulinemia precede the development of type II diabetes in offspring of diabetic parents. Ann Intern Med 113: 909-915

35. Saad MF, Knowler WC, Pettitt DJ, Nelson RG, Mott DM, Bennett PH (1989) Sequential changes in serum insulin concentration during development of non-insulin-dependent diabetes. Lancet I: 1356-1359

36. Saad MF, Knowler WC, Pettitt DJ, Nelson RG, Charles MA, Bennett PH (1991) A two-step model for development of non-insulin-dependent diabetes. Am J Med 90: 229-235

37. Welborn TA, Wearne K (1979) Coronary heart disease incidence and cardiovascular mortality in Busselton with reference to glucose and insulin concentrations. Diabetes Care 2: $154-160$

38. Eschwege E, Richard JL, Thibault N et al. (1985) Coronary heart disease mortality in relation with diabetes, blood glucose and plasma insulin levels: the Paris prospective study, ten years later. Horm Metab Res 15 [Suppl]: 41-46 
39. Modan M, Halkin H, Almong S et al. (1985) Hyperinsulinemia: a link between hypertension, obesity, and glucose intolerance. J Clin Invest 75: 809-817

40. Pyörälä K, Savolainen E, Kaukola S, Haapakoski J (1985) Plasma insulin as coronary heart disease risk factor: relationship to other risk factors and predictive value during 91/2year follow-up of the Helsinki policemen study population. Acta Med Scand 701 [Suppl]: 38-52

41. O'Sullivan JB, Mahan CM (1968) Prospective study of 352 young patients with chemical diabetes. New Engl J Med 278: 1038-1041

42. Jarrett RJ, Keen H, Fuller JH, McCartney M (1979) Worsening to diabetes in men with impaired glucose tolerance ("borderline diabetes"). Diabetologia 16: 25-30

43. Sasaki A, Suzuki T, Horiuchi N (1982) Development of diabetes in Japanese subjects with impaired glucose tolerance: a seven year follow-up study. Diabetologia 22: 154-157
44. Kadowaki T, Miyake Y, Hagura R et al. (1984) Risk factors for worsening to diabetes in subjects with impaired glucose tolerance. Diabetologia 26: 44-49

45. King H, Zimmet P, Raper LR, Baikau B (1984) The natural history of impaired glucose tolerance in the Micronesian population of Nauru: a 6-year follow-up study. Diabetologia 26: $39-43$

46. Stern MP, Rosenthal M, Haffner SM (1985) A new concept of impaired glucose tolerance: relation to cardiovascular risk. Arteriosclerosis 5: 311-314

47. Bennett PH (1985) Impaired glucose tolerance: a target for intervention? Arteriosclerosis 5: 315-317

48. Newell-Morris L, Fujimoto W, Grote M (1991) Risk factors for NIDDM and coronary heart disease in two generations of Japanese-American women. 14th IDF Congress Satellite, Epidemiology of diabetes and its complications, Williamsburg, VA (Abstract, No 68) 